\title{
Velocity-jump instabilities in Hele-Shaw flow of associating polymer solutions
}

\author{
D. H. Vlad, Jordi Ignes-Mullol* and J. V. Maher \\ Department of Physics and Astronomy, University of Pittsburgh, Pittsburgh, Pennsylvania 15260
}

(Received 19 March 1999; revised manuscript received 11 June 1999)

\begin{abstract}
We study fracturelike flow instabilities that arise when water is injected into a Hele-Shaw cell filled with aqueous solutions of associating polymers. We explore various polymer architectures, molecular weights, and solution concentrations. Simultaneous measurements of the finger tip velocity and of the pressure at the injection point allow us to describe the dynamics of the finger in terms of the "finger mobility," which relates the velocity to the pressure gradient. The flow discontinuities, characterized by jumps in the finger tip velocity, which are observed in experiments with some of the polymer solutions, can be modeled by using a nonmonotonic dependence between a characteristic shear stress and the shear rate at the tip of the finger. A simple model, which is based on a viscosity function containing both a Newtonian and a non-Newtonian component, and which predicts nonmonotonic regions when the non-Newtonian component of the viscosity dominates, is shown to agree with the experimental data. [S1063-651X(99)08910-2]

PACS number(s): 47.50.+d, 47.20.Gv, 47.20.Ma, 47.54.+r
\end{abstract}

\section{INTRODUCTION}

The Saffman-Taylor instability [1], which arises when a less viscous fluid invades a more viscous fluid confined between closely spaced parallel plates, has become the model of choice in studies on many pattern formation systems, due to its relative simplicity, its analogy with the MullinsSekerka instability at the solidification front [2], and its application to the study of flow through porous media, crude oil recovery, and air-assisted injection molding. The dynamics of the viscous fingers observed in the flow of Newtonian fluids confined to a narrow gap between two parallel rigid walls (Hele-Shaw cell) is now well understood [1]. The basic transport equation for the gap averaged flow is Darcy's law:

$$
\mathbf{u}=-\frac{b^{2}}{12 \mu} \nabla \mathbf{P}=-\mathcal{M} \nabla \mathbf{P}
$$

where $\mathbf{u}$ is the gap-averaged velocity, $b$ is the gap size, $\mu$ is the viscosity, $P$ is the pressure, and $\mathcal{M}$ is the mobility. For Newtonian fluids, in the case of a nonzero surface tension at the interface, the width of the finger is unique [3] and it has been measured experimentally [1]. The dimensionless parameter $\lambda$, which represents the relative width of the finger compared to the width of the channel, is a decreasing function of the dimensionless parameter $1 / B=12(\mu \mathrm{V} / \sigma)(w /$ $b)^{2}$, and reaches values close to $\frac{1}{2}$ in the regime of large velocities, where capillary forces are dominated by viscous forces.

Displacement processes in rheologically complex fluids like homopolymer solutions [4], clay dispersions [5], foams [6], and associating polymers [7,8] lead to a very wide variety of patterns. The non-Newtonian behavior of these fluids-viscoelasticity, shear thinning and thickening, yield stress etc.-adds complexity and apparent nonuniversality to the patterns.

\footnotetext{
*Present address: Department of Chemistry, Tulane University, New Orleans, LA 70118.
}

Previous experiments using linear associating polymers in radial [7] and channel [8] geometry have shown a transition from a viscous fingering regime to a fracturing pattern. The transition was shown to occur when the injection rate becomes higher than a threshold value. The lower molecular weight (MW) linear polymers (MW 17500 and 51000 ) exhibit an abrupt increase in tip velocity followed by a much milder increase in velocity after the transition. The highest molecular weight polymer (MW 100400) shows a significantly different behavior: a smooth transition to a regime characterized by high fluctuations (dominated by a characteristic frequency that itself depends linearly on volumetric injection rate [8]) in the tip velocity followed by another smooth transition to a fracturelike regime.

Patterns resembling those in associating polymer solutions were observed in experiments in which nitrogen at constant pressure was injected into surfactant-brine mixtures $[9,10]$. At low salt concentrations, in the spherulite phase, ramified fingers were obtained. At a higher salt concentration, the spherulite phase turns into a lamellar phase, and the viscous finger shows a transition to a narrow needle, which resembles the fracturelike finger in associating polymer solutions. The relative width $\lambda$ of the finger was found to decrease to zero at a finite time after the flow was established, while the finger tip velocity increased dramatically in time, reminiscent of a "finite-time singularity." At even higher salinity, in the sponge phase the finger is normal, although its relative width decreases below $\frac{1}{2}$.

The surfactant-brine mixture exhibits extreme shear thinning and a stress plateau that can lead to a bistable flow [10]. It was pointed out that the appearance of the fracturelike instabilities might be connected to the flow bistability.

None of the experiments in associating polymer solutions $[7,8]$ or surfactant-brine mixtures $[9,10]$ provided measurements of the stress inside the polymer solution during the unstable finger growth.

In the present work, we describe the rheological characteristics of the fracturelike instabilities in associating polymer solutions by simultaneous measurements of the shear 


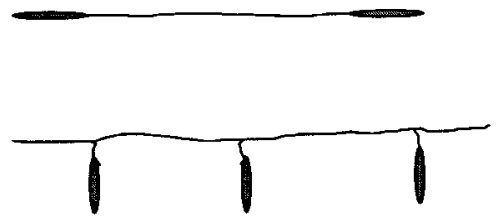

(a)

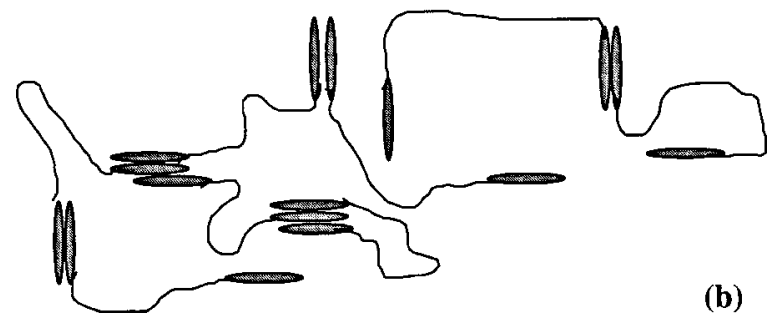

FIG. 1. (a) A sketch of the associating polymer: linear endcapped and comb architectures. (b) Associating network in a polymer solution.

stress and shear rate during the unstable viscous fingering experiments, while observing the changing morphology of the patterns.

\section{DESCRIPTION OF THE ASSOCIATING POLYMER SOLUTIONS}

The associating polymers [11] used in Refs. [7] and [8] and in the present experiments are amphiphilic molecules consisting of a linear hydrophilic backbone [polyethyleneglycol (PEG), chain-extended by di-isocyanates] and hydrophobes (C16 alkanols) attached to the backbone. In this paper we report the results of measurements of solutions using two distinct polymer architectures: linear end-capped molecules, of molecular weights 17500,51000 , and 100400 (the same polymers as those used in Refs. [7, 8]), and comb polymers of molecular weights 53300, 106800, and 160300 . A sketch of the polymer molecule architectures is shown in Fig. 1(a).

In aqueous solutions, the hydrophobes try to minimize contact with water and in that process they create an associative three-dimensional (3D) network [see Fig. 1(b)], which confers viscoelasticity to the polymer solutions $[11,12]$. However, the binding energy of a hydrophobe in a micelle is small enough to allow hydrophobes to disengage at random at a finite rate, and therefore the network bonds are not rigid and associating polymer solutions do not turn into gels $[11,12]$. Rheological measurements observed no yield stress in these fluids $[11,12]$.

In shear flow, the associating polymer solutions show a fairly constant viscosity at small shear rate. As the shear rate is increased, the viscosity first increases by a small amount and then decreases dramatically for shear rates larger than a characteristic shear-thinning shear rate $\dot{\gamma}_{0}[11,12]$.

The shear-thinning regime can extend over few shear rate decades, and is steeper for the more concentrated solutions. As observed from the viscosity measurements in [11], solutions having the largest hydrophobes concentration (high polymer mass concentration and high fraction of hydrophobes per backbone unit length) show a local stress plateau characterized by a slope of the viscosity vs shear rate steeper than -1 on a log-log scale. In these regions the shear stress decreases with the shear rate. Less concentrated solutions generally show only shear thinning, sometimes very strong, but they show no stress plateau.

The polymer solutions are viscoelastic. The relaxation time, defined as the inverse of the frequency at which the storage modulus become equal to the loss modulus, is of the order of $0.1 \mathrm{sec}$ or less for the linear end-capped polymers and for the low molecular weight comb polymers [11]. The comb polymers of high molecular weight have a much larger relaxation time, of the order of many seconds [13].

\section{EXPERIMENTAL OBSERVATIONS}

In our experiments we injected dyed water at constant volumetric flow rate into a Hele-Shaw channel of length $L$ $=22.5 \mathrm{~cm}$, width $w=1 \mathrm{~cm}$, and gap between the plates $b$ $=0.15 \mathrm{~cm}$, filled with associating polymer solutions of various concentrations. Flow realizations were recorded on videotape, and the crack-tip position and the area of the pattern were subsequently extracted by using image-processing software packages. The velocity of the tip, $\mathrm{v}_{f}$, was constructed from the tip position. The effective width of the finger was calculated from the increase of the area $A$ in time:

$$
w_{f}=\frac{1}{\mathrm{v}_{f}} \frac{d A}{d t} \text {. }
$$

Since the width of the finger behind the advancing tip does not change in time, $w_{f}$ provides a good measure for the actual finger width.

The pressure of the dyed water at the injection point was measured by using pressure transducers. Since the viscosity of the water is much smaller than the viscosity of the polymer solution, the pressure drop within the dyed water finger can be neglected, compared to the pressure drop due to the shear of the polymer solution ahead of the finger. Thus, the pressure recorded at the injection point is assumed to be approximately the same as the pressure at the finger tip.

Several types of patterns were observed. In experiments with high molecular weight (MW 106800 and 160300) comb polymers, the dyed water injected into the channel formed a very disordered pattern along one of the lateral walls of the channel, as in Fig. 2, and viscous fingers were not obtained even at very low flow rates.

Steady-state viscous fingers were observed at low injection rates for all linear end-capped polymers and for the comb polymer of molecular weight 53300 . At higher injection rate the viscous finger became unstable and we observed three main types of patterns. For the linear end-capped polymer of molecular weight 100400 , in the entire concentration range we studied, 3-8\%, and for the comb polymer of molecular weight 53300 and concentrations $1.58 \%$ and $1.83 \%$, the viscous fingering regime continues with a "meandering", regime, characterized by tip oscillations along the channel with a characteristic frequency described in [8]. As the injection rate increases (injection rate is held constant in any one flow realization), the finger tip velocity increases continuously, while the fingers become narrower, with $\lambda=w_{f} / w$, the relative finger width, decreasing below $\frac{1}{2}$. Then the finger enters a fast fracture regime, of very rapidly increasing tip 


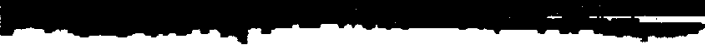

FIG. 2. Pattern obtained for an aqueous solution of comb associating polymer of $\mathrm{MW}=106800$ in concentration $1.06 \%$. The injection rate was $Q=0.1 \mathrm{ml} / \mathrm{min}$.

velocity and very narrow fingers. In this regime the tip oscillations cease and the finger grows centered along the channel.

The pressure shows an initial increase, possibly due to the stress building up in the polymer network as the polymer chains start stretching in the shear flow, or possibly, due to a lack of constancy of the flow rate at the beginning of the run. After it reaches a local maximum value, the pressure decreases as the finger moves toward the open end of the cell. A typical pattern, accompanied by the relative finger width, tip speed, and pressure for this class of behavior is shown in Fig. 3. The example corresponds to a $4.4 \%$ solution of linear end-capped polymer of molecular weight 100400 and an injection rate of $Q=4 \mathrm{ml} / \mathrm{min}$.

The dynamics of the fingers in solutions of linear endcapped polymers of molecular weight 17500 and 51000 present noticeable departures from the dynamics described above. The flow starts with a Saffman-Taylor finger but the transition to the fast-fracture regime is very abrupt (less than $\frac{1}{30} \mathrm{sec}$, the time resolution of our video recording). The finger tip velocity and the finger width are discontinuous at the onset of the fracturelike instability. After the jump, the finger tip velocity continues increasing, until it reaches a plateau value toward the end of the cell. The finger thickness decreases considerably, as seen from the change in the relative transparency of the finger.

When the injection rate is large enough, a new instability follows the fracturelike regime. The finger slows down rapidly and expands laterally until it reaches the sides of the channel. The tip of the finger becomes more pointed than a classical Saffman-Taylor finger. The transition is not instantaneous; it occurs in a time interval of the order of $0.2 \mathrm{sec}$. A sample example for this class of patterns is shown in Fig. 4, for a flow rate of $Q=2.5 \mathrm{ml} / \mathrm{min}$ and a linear end-capped polymer of molecular weight 51000 in concentration $2.5 \%$.

Solutions of comb polymer of MW 53300 in concentrations $2.55 \%$ and $3 \%$ combine the features of both classes of patterns described above. Figure 5 shows the pattern, width, and finger speed for a 3\% solution and an injection rate of $Q=1.18 \mathrm{ml} / \mathrm{min}$. The smooth viscous finger is followed by a meandering pattern and is continued by a fracture regime that starts with jumps in velocity and finger width.

\section{RHEOLOGICAL CHARACTERISTICS OF FRACTURE INSTABILITIES}

As the previous examples have shown, fracture and meandering instabilities appear to be rather independent of one another in the Saffman-Taylor flow. It is even possible that the origin of those two instabilities resides in two different rheological properties of the polymer solutions. The best candidates are the strong shear thinning and the viscoelasticity of the solutions. We will use our pressure and velocity measurements to estimate the stress and the shear rate at the
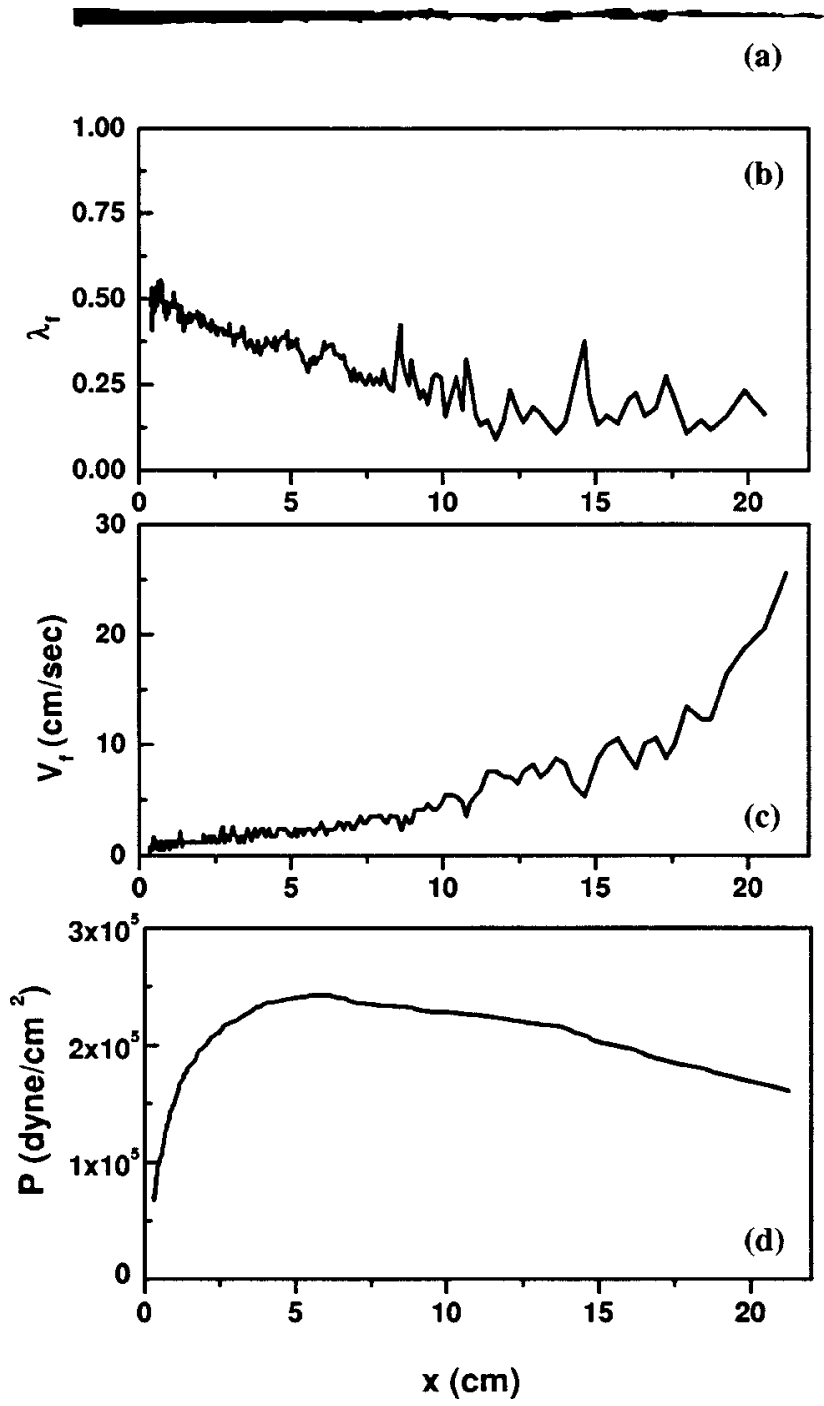

FIG. 3. (a) Pattern obtained for a solution of linear end-capped associating polymer of $\mathrm{MW}=100400$ and $c=4.4 \%$, (b) finger width relative to the width of the channel, (c) finger tip velocity, and (d) pressure at the cell entrance. $Q=4 \mathrm{ml} / \mathrm{min}$.

tip of the finger in order to infer some dynamical characteristics of the fracture instabilities.

\section{A. Stress-velocity diagram}

The velocity data can be used to infer a measure for the shear rate in the polymer solution near the tip of the finger. The smallest length scale that constrains the flow is the cell thickness $b$. The liquid at the center of the gap moves at the interface velocity and, assuming no slip at the glass plates boundary, we obtain a relevant shear rate at the tip of the finger,

$$
k_{f}=\mathrm{v}_{f} / b^{\prime}
$$

with $b^{\prime}=b / 2$.

The stress tensor elements in the neighborhood of the tip of the finger have a very complex dependence on a large number of parameters, e.g., location relative to the moving interface, injection rate, channel geometry, rheological properties of the solution, etc. Still, a measure of the shear stress 
(a)

(b)
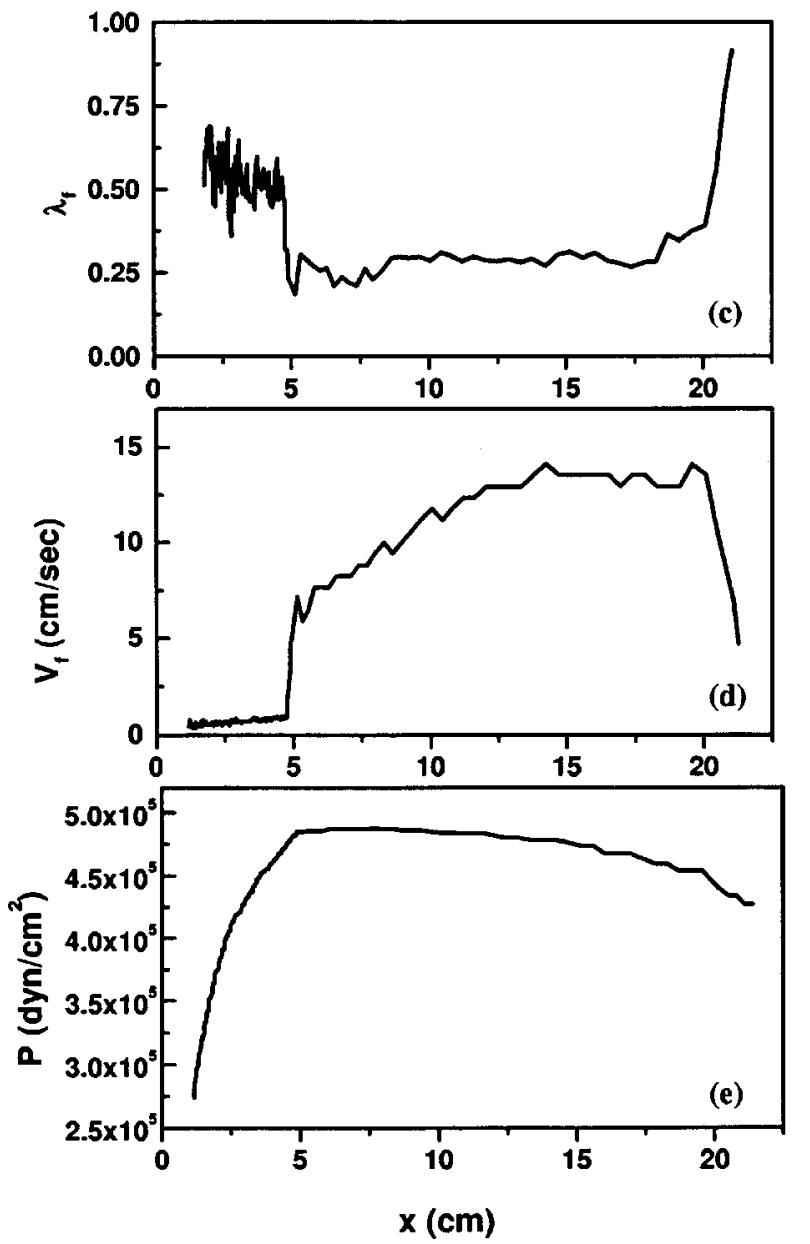

FIG. 4. (a) and (b) Two images of the pattern obtained in an experiment with a solution of linear polymer of $\mathrm{MW}=51000$ in concentration $2.5 \%$, (c) relative finger width, (d) velocity of the finger, and (e) pressure at the injection point. $Q=2.5 \mathrm{ml} / \mathrm{min}$.

inside the polymer solution, ahead of the finger, can be obtained from the pressure measurements:

$$
\sigma=|\nabla P| b^{\prime} .
$$

In the previous equation the absolute value of the pressure gradient was used since in shear flow the pressure gradient is negative.

A plot of the pressure gradient in the polymer solution vs the finger tip velocity for the linear end-capped polymers of MW 100400 and $c=4.4 \%$ is shown in Fig. 6 and for MW 51000 and $c=2.5 \%$ in Fig. 7. The pressure gradient was calculated by dividing the pressure of the invading fluid by the length of the polymer region in front of the finger. To minimize the effects associated with the open end of the cell, we considered only the data for which the tip of the finger is more than $3 \mathrm{~cm}$ from the open end of the cell. For the examples displayed in Fig. 7, the data show the fingering and fracturelike regimes and not the regime observed towards the end of the experiment when the finger fills the entire channel width.

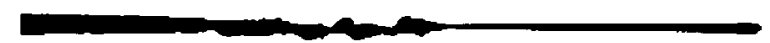

(a)

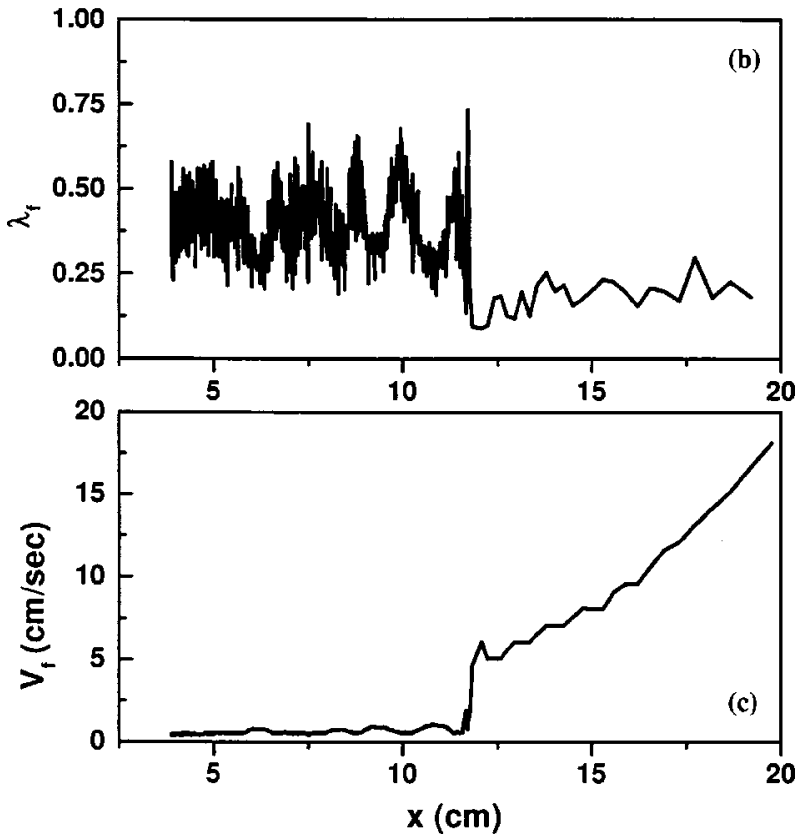

FIG. 5. (a) Pattern in an experiment with a solution of comb associating polymer of MW $=53300$ and $c=3 \%$, (b) relative finger width, and (c) finger tip velocity. The injection rate is $1.18 \mathrm{ml} / \mathrm{min}$.

The first observation is that for each polymer solution all points from experiments performed at different injection rates fall on the same curve. The existence of a unique curve suggests the absence of significant memory effects, associated with viscoelasticity of the solutions. The relaxation times of the polymer solutions are known to be relatively small (of the order of $0.1 \mathrm{sec}$ in the examples shown in Figs. 6 and 7) [11], much smaller than the time of the total flow (of several seconds up to a few minutes, depending on the injection rate). Viscoelastic effects might play an important

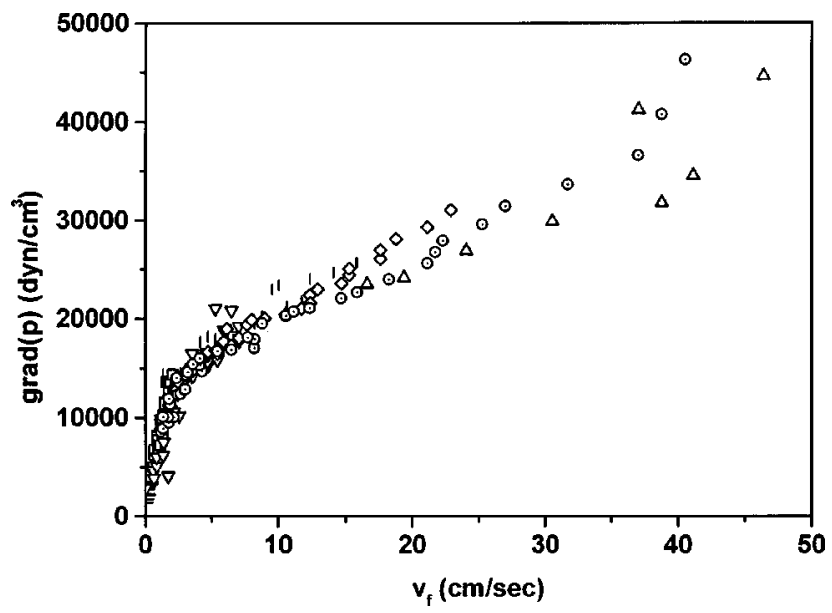

FIG. 6. Pressure gradient vs finger tip velocity for a solution of linear end-capped associating polymer of $\mathrm{MW}=100400$ and $c$ $=4.4 \%$. The experiments are performed at different injection rates: -, $Q=1 \mathrm{ml} / \mathrm{min} ; \quad \square, Q=3 \mathrm{ml} / \mathrm{min} ; \quad \nabla, Q=4 \mathrm{ml} / \mathrm{min} ; \quad \mid, \quad Q$ $=5 \mathrm{ml} / \mathrm{min} ; \quad \diamond, \quad Q=6 \mathrm{ml} / \mathrm{min} ; \quad \odot, \quad Q=8 \mathrm{ml} / \mathrm{min} ; \triangle, \quad Q$ $=18 \mathrm{ml} / \mathrm{min}$. 
role in the immediate vicinity of the finger tip, where the flow could suffer important changes over periods of time shorter than the relaxation time of the polymer solutions, but their presence in the pressure data should probably be masked by the pressure drop within the column of polymer in front of the finger.

\section{B. Finger mobility}

Since the relation between finger tip velocity and pressure gradient is observed to be unique for each solution under study, we can define a finger mobility $\mathcal{M}_{f}$,

$$
\mathbf{v}_{f}=-\mathcal{M}_{f} \nabla \mathbf{P}
$$

$\mathcal{M}_{f}$ thus describes the dynamics of the finger in the HeleShaw cell and it is expected to be strongly dependent on the rheological properties of the liquid.

A relation analogous to the Darcy law was written in Ref. [14] for nonlinear viscous fluids,

$$
\mathbf{u}=-\frac{b^{2}}{12 \bar{\mu}} \nabla \mathbf{P},
$$

where $\bar{\mu}$ does not represent the true viscosity, but rather a gap-averaged viscosity function. It was shown that usually there is a direct relation between the monotony of $\bar{\mu}$ and that of the true viscosity function. A shear thinning behavior, for example, will correspond to a decreasing $\bar{\mu}$. For a Newtonian fluid $\bar{\mu}$ is equal to the constant viscosity of the fluid.

Equation (6) can be used to infer an effective (gapaveraged) viscosity for the fluid in the Hele-Shaw cell from the slope of the pressure gradient vs the gap-averaged velocity in the channel. The quantity observed in pattern formation experiments is the finger tip velocity, which is different from the average velocity in the channel. A simplified approach, in which the gap-averaged fluid velocity was substituted with the finger tip velocity in Eq. (6) was used in Ref. [15] to determine an "effective viscosity."

A more rigorous approach has to relate the finger tip velocity to the gap-averaged velocity of the polymeric fluid inside the channel. A plausible relation between the velocities can be inferred as follows. The injection rate $Q$ can be written as the flow rate in the water finger of velocity $\mathbf{v}_{f}$ and cross-sectional area $\Sigma_{f}$, and also as the flow rate in the polymer solution region in front of the finger, of average velocity $u$ and cross-sectional area equal to that of the channel, $\Sigma_{0}$ $=b w$,

$$
Q=\mathrm{v}_{f} \Sigma_{f}=u \Sigma_{0}
$$

Therefore,

$$
\mathrm{v}_{f}=u \frac{\Sigma_{0}}{\Sigma_{f}} .
$$

$\Sigma_{0} / \Sigma_{f}$ is equal to $1 / \lambda$ when neglecting the layer of fluid left on the walls behind the moving interface, and $u=\lambda \mathrm{v}_{f}$ (see also Ref. [9]). The finger mobility can then be written as

$$
\mathcal{M}_{f}=\frac{b^{2}}{12 \bar{\mu}} \frac{\Sigma_{0}}{\Sigma_{f}} .
$$

Equation (9) provides a more rigorous method for determining the effective viscosity $\bar{\mu}$ of the non-Newtonian fluid in the Hele-Shaw cell. For a Newtonian fluid $\bar{\mu}$ is constant and represents the true viscosity of the fluid, $\mu$. At large capillary numbers $\lambda$ reaches a limiting value of $\frac{1}{2}$, and it can be considered to be approximately constant. With these assumptions, the finger mobility for a Newtonian fluid is also approximately constant:

$$
\mathcal{M}_{f}=\frac{b^{2}}{12 \mu \lambda} .
$$

In Figs. 6 and 7 the low velocity (shear rate) and low pressure gradient (shear stress) region corresponds to the Saffman-Taylor viscous fingering regime. Since the viscosity of each polymer solution is fairly constant at low shear rate [11], in this regime the polymer solution behaves like a Newtonian fluid with a viscosity $\mu_{0}$. The tip velocity is proportional to the pressure gradient and the finger mobility is constant. For the linear end-capped polymer of MW 100400 of $c=4.4 \%$, the mobility at low shear rate, obtained as the inverse of the slope of the linear regime in Fig. 6 is equal to $1.2 \pm 0.2 \times 10^{-4} \mathrm{~cm}^{3} \mathrm{sec} / \mathrm{g}$. This value is in agreement with the value obtained from Eq. (10), $\mathcal{M}_{f}=1.0 \pm 0.2$ $\times 10^{-4} \mathrm{~cm}^{3} \mathrm{sec} / \mathrm{g}$, for $\lambda=\frac{1}{2}$ and a low shear rate constant viscosity $\mu_{0}=38 \pm 6 \mathrm{P}$. The value for $\mu_{0}$ used in the calculation was obtained by interpolating the measured dependence of $\mu_{0}$ on concentration [11] at $c=4.4 \%$.

Similarly, the measured mobility at low shear rate for the polymer solution in Fig. 7 is $3.3 \pm 0.5 \times 10^{-5} \mathrm{~cm}^{3} \mathrm{sec} / \mathrm{g}$, while the calculated mobility is $2.9 \times 10^{-5} \mathrm{~cm}^{3} \mathrm{sec} / \mathrm{g}$, for a low shear rate viscosity $\mu_{0}=129 \mathrm{P}$, reported in [11].

In both Figs. 6 and 7, at high tip velocity the relation between the pressure gradient and tip velocity is also linear, however, with a much smaller slope. This region corresponds to the fast fracture of the polymer solutions. The presence of a smaller slope at high tip velocity is consistent with the known shear-thinning behavior of the solutions.

The transition between the viscous fingering and fast fracture is different in Figs. 6 and 7. For the linear end-capped polymer of MW 100400 the transition is continuous. The region where the slope turns can be roughly identified with the meandering regime in the dynamics of the finger. For the polymer of MW 51000 the transition is abrupt, and the local pressure gradient is very flat. In all cases, the transitions occur for values of the shear rate at the tip of the finger in agreement with the values for the shear thinning shear rate from Ref. [11].

\section{A simple model for flow discontinuity}

The appearance of the flow discontinuity in finger growth can be modeled with a nonmonotonic dependence between the shear stress and the shear rate. A simple model that can incorporate this feature is

$$
\sigma=\left(\frac{\eta_{1}}{1+\alpha \tau^{2} k_{f}^{2}}+\eta_{2}\right) k_{f},
$$

where $\eta_{2}$ is a constant Newtonian viscosity and $\eta_{1}$ determines the strength of the non-Newtonian effects. $\tau$ is the relaxation time of the liquid and $\alpha$ is a numerical constant. 


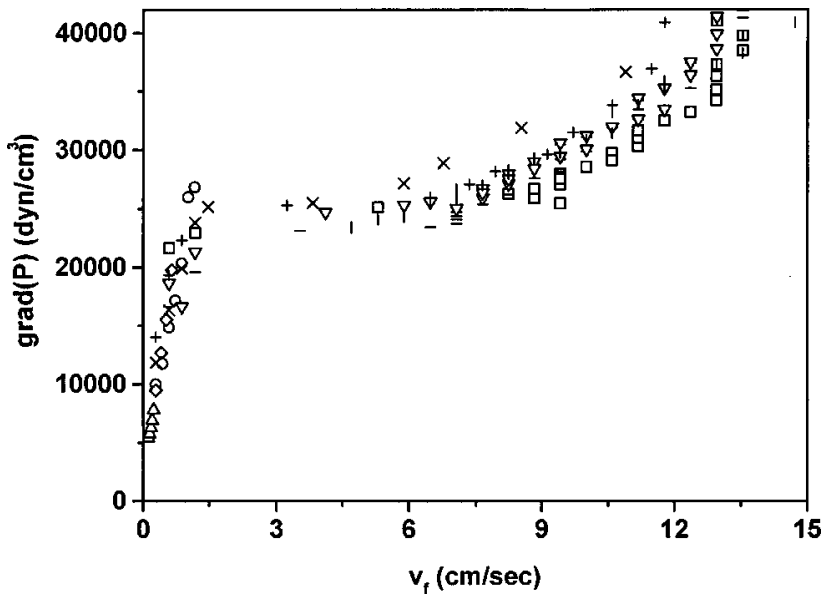

FIG. 7. Pressure gradient vs finger tip velocity for a solution of linear associating polymer of $\mathrm{MW}=51000$ and $c=2.5 \%$. The injection rates are as follows: $\triangle, Q=0.5 \mathrm{ml} / \mathrm{min} ; \diamond, Q=1 \mathrm{ml} / \mathrm{min}$; ,$+ Q=1.5 \mathrm{ml} / \mathrm{min} ; \times, Q=2 \mathrm{ml} / \mathrm{min} ; O, Q=2 \mathrm{ml} / \mathrm{min} ; \nabla, Q$ $=2.5 \mathrm{ml} / \mathrm{min} ; \quad-, \quad Q=3 \mathrm{ml} / \mathrm{min} ; \quad \square, \quad Q=4 \mathrm{ml} / \mathrm{min} ; \quad \mid, \quad Q$ $=6 \mathrm{ml} / \mathrm{min}$.

At small shear rate the relationship between stress and shear rate is linear with the slope $\eta_{1}+\eta_{2}$ and the dynamics is Newtonian. At larger shear rate the viscosity decreases and approaches $\eta_{2}$ in the limit of high shear rate. A shear-stressshear-rate dependence similar to that in Eq. (11) is widely used in the description of flow of Johnson-Segalman fluids in viscometric flow [16], and was used in Ref. [17] to explain the "spurt" instabilities observed in the flow of viscoelastic polymer melts through capilaries [18] and variations of this model were used in the study of Hele-Shaw flow $[14,19]$. The relationship between the pressure gradient and finger tip velocity and implicitly the finger mobility can be obtained by combining Eqs. (3), (4), and (11),

$$
|\nabla P|=\frac{\eta_{2}}{b^{\prime 2}}\left(\frac{\eta_{1} / \eta_{2}}{1+\frac{\alpha \tau^{2}}{b^{\prime 2}} \mathrm{v}_{f}^{2}}+1\right) \mathrm{v}_{f}=\frac{1}{\mathcal{M}_{f}} \mathrm{v}_{f}
$$

It should be emphasized that the relation in Eq. (11) is not equivalent to the constitutive relation for the polymer solution, and therefore $\mathcal{M}_{f}$ cannot be determined from pure rheological measurements. Rheological properties of the polymer solutions connect the pressure gradient to the gap-averaged flow velocity in the channel and not to the finger tip velocity.

When the non-Newtonian term dominates, for $\eta_{1} / \eta_{2}$ $>8$, the model predicts a non-monotonic dependence between the pressure gradient and the finger tip velocity. This feature of the model is determined only by $\eta_{1} / \eta_{2}$, and it does not depend on the values of the other model parameters, $\alpha$ and $\tau$. The characteristics of the model are qualitatively illustrated in Fig. 8. Applying this model to the fingering problem, a nonmonotonic $\nabla P$ vs $\mathrm{v}_{f}$ will result in finger tip velocity jumps. In the viscous fingering regime, as $\nabla P$ increases, the finger tip velocity will increase accordingly. When the local maximum for $\nabla P$ is reached, the velocity will jump from the low $\nabla P$ and low velocity branch to the high $\nabla P$ and high velocity stable branch. When the Newton-
TABLE I. Comparison between the shear stress vs shear rate diagram obtained experimentally for several polymer solutions and the model in Eq. (12). The parameter $\eta_{1} / \eta_{2}$ is determined by comparing the slope of the diagram at low shear rate with the slope at high shear rate.

\begin{tabular}{ccllr}
\hline \hline $\begin{array}{c}\text { Polymer } \\
\text { architecture }\end{array}$ & MW & $\begin{array}{c}c \\
(\%)\end{array}$ & $\begin{array}{c}\text { Tip } \\
\text { velocity }\end{array}$ & $\eta_{1} / \eta_{2}$ \\
\hline comb & 53300 & 3 & discontinuous & $16 \pm 3$ \\
linear & 50700 & 2.5 & discontinuous & $16 \pm 4$ \\
comb & 53300 & 2.55 & discontinuous & $12 \pm 2$ \\
linear & 100400 & 4.4 & continuous & $9 \pm 2$ \\
linear & 100400 & 3 & continuous & $6 \pm 1$ \\
comb & 53300 & 1.58 & continuous & $4 \pm 1$ \\
\hline \hline
\end{tabular}

ian viscosity dominates, there are no nonmonotonic regions in the pressure gradient-velocity diagram, and therefore no jumps in the finger velocity.

This simple model is consistent with the experimental data. The model viscosity parameters $\eta_{1}$ and $\eta_{2}$ can be obtained from the measured values of $\nabla P$ vs $\mathrm{v}_{f}$, from the slope at low shear rate and at high shear rate. The other parameters can be determined from the shape of the intermediate regime, but they are not relevant for our analysis since, as mentioned before, they do not determine the appearance of the nonmonotonic region in the $\nabla P$ vs $\mathrm{v}_{f}$ diagram. Table I summarizes the comparison with the model. As the model predicts, velocity jumps are observed when $\eta_{1} / \eta_{2}$ is higher than a threshold value, i.e., when the non-Newtonian viscosity dominates. The value of the threshold observed experimentally is not inconsistent with the threshold predicted by the model, approximately 8 , accounting for uncertainties in the determination of $\eta_{1} / \eta_{2}$.

\section{Comments on the meandering regime}

The high complexity of the meandering regime prevents us from offering a satisfactory interpretation for its origin.

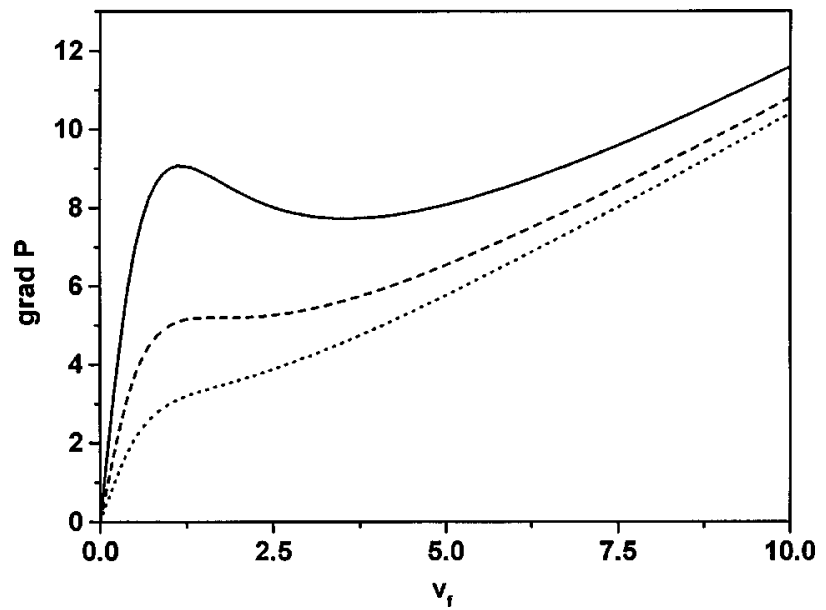

FIG. 8. Pressure gradient vs finger tip velocity curves predicted by the model in Eq. (12) for three different values of $\eta_{1}$. For simplicity we chose $\eta_{2}=\alpha=\tau=b^{\prime}=1$. The continuous line corresponds to $\eta_{1} / \eta_{2}=16$, the dashed line to $\eta_{1} / \eta_{2}=8$, and the dotted line to $\eta_{1} / \eta_{2}=4$. 
However, some arguments and experimental studies [20] suggest that the meandering regime might be determined, or at least influenced, by the viscoelastic properties of the solutions. In the flow of viscoelastic fluids, the stress tensor is no longer isotropic as in the case of Newtonian fluids. Normal stress components arise due to the stretching of the polymer chains in shear flow. Most constitutive equations for nonNewtonian fluids predict that the normal stress components increase with the second power of the shear rate [16], faster than shear stress, which is proportional to shear rate. Therefore, the normal stresses, which should not significantly influence the dynamics of the finger at low velocity, might strongly influence the finger dynamics at high velocity. Additionally, viscoelastic fluids possess a "fading memory," and in time-dependent flow (as for the flow near the tip of the finger) they are known to exhibit a complex transitory response, e.g., stress growth and stress relaxation [21]. Recent theoretical and experimental studies [22] show that the viscoelasticity can impact significantly the viscous fingering problem, e.g., changing the thickness of the film left behind the finger on the glass plates.

The normal stress components and the fading memory of the fluids change the stress distribution in the neighborhood of the tip of the finger, and they might disturb the finger from its straight path along the center of the channel [20].

The patterns obtained with the higher molecular weight comb polymers might be an extreme case of finger meandering. Even when using very small injection rates, we observed that the flow occurred only along the walls. In contrast with the other associating polymer solutions studied, which have a rather small relaxation time (about $0.1 \mathrm{sec}$ or shorter), the higher molecular weight comb polymers solutions studied have relaxation times in excess of $15 \mathrm{sec}$ [13], which makes them strongly viscoelastic.

\section{STABILIZING EFFECT OF THE SURFACE TENSION}

All the experiments reported in the previous sections were carried on by injecting dyed water into the polymer solutions. The interfacial tension between the aqueous polymer solution and the dyed water should be very small, although not zero, being determined mainly by the concentration gradients that exist at the interface between the fluids. An effective interfacial tension, determined by the excess free energy at the interface due to concentration gradients, can be defined even for a dissolving interface between miscible liquids [23].

Most of the theoretical studies of the viscous fingering problem assume a nonzero surface tension. Surface tension enters the description of the fingers through the capillary number $1 / B=12(\mu \mathrm{V} / \sigma)(w / b)^{2}$. Since the interfacial tension at the water-polymer solution is very small, the experiments reported above describe the high capillary number limit.

To investigate the effect of a much larger interfacial tension on the onset of the fracturelike instabilities, we carried out experiments in which we injected air at constant pressure into two solutions of comb associating polymer of MW 53300 of concentrations $1.58 \%$ and $2.55 \%$.

In these experiments we produced the same instabilities as those obtained at the dyed water-polymer solution interface. However, certain noticeable differences were observed. The shape of the tip of the finger at the air-polymer solution

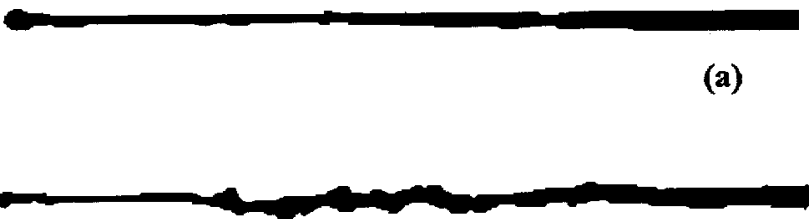

(b)

FIG. 9. Air injected into comb associating polymer (MW 53300 ) solutions. (a) $c=1.58 \%$ and (b) $c=2.52 \%$.

interface is more rounded, and the tip is wider than the body of the finger (see Fig. 9).

Using the same image acquisition techniques as those described above, we extracted the finger tip velocity at the onset of the instabilities, and we compared the results with the results from experiments in which dyed water was used as the injection agent. The results are presented in Table II. All data present a significant shift of the instabilities toward higher velocities, showing the stabilizing effect of the interfacial tension.

\section{CONCLUSIONS}

Experimental observations of the flow instabilities occurring in the Saffman-Taylor flow of associating polymer solutions show a variety of features, e.g., finger meandering, fast-fracture, and velocity jumps. To understand the common characteristics of these instabilities, we studied several solutions, of different concentration, polymer molecular weight, and architecture. Although the resulting pattern is highly dependent on the type of polymer solution, a plot of the finger tip velocity (a measure for the shear rate at the tip of the finger) vs pressure gradient (a measure for the shear stress) reveals interesting similarities. For each solution, the data from all experiments performed at different flow rate realizations follow the same master curve. At low tip velocity, in the viscous fingering regime, the pressure gradient is proportional with the finger tip velocity. As the velocity increases, the slope of the curve decreases, due to the shear-thinning properties of the solutions.

The flow discontinuities, characterized by jumps in finger tip velocity, observed in experiments with some of the polymer solutions, were modeled with a nonmonotonic depen-

TABLE II. The finger tip velocity at the onset of the meandering and fracture instabilities in the absence of the interfacial tension (water-polymer solution interface) and for a nonzero interfacial tension (air-polymer interface). Two solutions of different concentrations of comb associating polymer with a molecular weight of MW 53300 were used.

\begin{tabular}{lccc}
\hline \hline $\begin{array}{l}c \\
(\%)\end{array}$ & $\begin{array}{c}\text { Injection } \\
\text { agent }\end{array}$ & $\begin{array}{c}\text { Tip velocity at the } \\
\text { meandering instability } \\
(\mathrm{cm} / \mathrm{sec})\end{array}$ & $\begin{array}{c}\text { Tip velocity at the } \\
\text { fracture instability } \\
(\mathrm{cm} / \mathrm{sec})\end{array}$ \\
\hline 1.58 & air & $7.3 \pm 0.5$ & $23.1 \pm 1.5$ \\
1.58 & dyed water & $3.0 \pm 0.3$ & $12.3 \pm 1.1$ \\
2.55 & air & $1.56 \pm 0.13$ & $4.23 \pm 2.49$ \\
2.55 & dyed water & $0.71 \pm 0.18$ & $1.25 \pm 0.22$ \\
\hline \hline
\end{tabular}


dence between velocity and shear stress. In this model the viscosity function has both a Newtonian and a nonNewtonian component, and flow discontinuities occur when the non-Newtonian term dominates. The experimental data show that the model is successful in describing the appearance of the flow discontinuities.

\section{ACKNOWLEDGMENTS}

We wish to thank Anne Robertson for helpful discussions and A. Baratt for help with data acquisition. This work was supported by the U.S. DOE under Grant No. DE-FG0284ER45131.
[1] P. G. Saffman and G. I. Taylor, Proc. R. Soc. London, Ser. A 245, 312 (1958); D. Bensimon, L. P. Kadanoff, S. Liang, B. I. Shraiman, and C. Tang, Rev. Mod. Phys. 58, 977 (1986); G. M. Homsy, Annu. Rev. Fluid Mech. 19, 271 (1987).

[2] W. W. Mullins and R. F. Sekerka, J. Appl. Phys. 35, 444 (1964).

[3] J. W. McLean and P. G. Saffman, J. Fluid Mech. 102, 455 (1981); R. Combescot, T. Dombre, V. Hakim, Y. Pomeau, and A. Pumir, Phys. Rev. Lett. 56, 2036 (1986); B. I. Shraiman ibid. 56, 2028 (1986); D. C. Hong and J. Langer, ibid. 56, 2032 (1986).

[4] J. Nittman, G. Daccord, and H. E. Stanley, Nature (London) 314, 141 (1985); G. Daccord, J. Nittman, and H. E. Stanley, Phys. Rev. Lett. 56, 336 (1986); H. Zhao and J. V. Maher, Phys. Rev. A 45, R8328 (1992).

[5] H. Van Damme, C. Laroche, L. Gatineau, and P. Levitz, J. Phys. (France) 48, 1121 (1987); H. Van Damme, E. Alsac, C. Laroche, and L. Gatineau, Europhys. Lett. 5, 25 (1988); E. Lamaire, P. Levitz, G. Daccord, and H. Van Damme, Phys. Rev. Lett. 67, 2009 (1991).

[6] S. Park and D. Durian, Phys. Rev. Lett. 72, 3347 (1994).

[7] H. Zhao and J. V. Maher, Phys. Rev. E 47, 4278 (1993).

[8] Jordi Ignes-Mullol, H. Zhao, and J. V. Maher, Phys. Rev. E 51, 1338 (1995).

[9] O. Greffier, A. Al kahwaji, J. Rouch, and H. Kellay, Phys. Rev. Lett. 81, 3860 (1998).

[10] D. Bonn, H. Kellay, and J. Meunier, Philos. Mag. B 78, 131 (1998).

[11] R. D. Jenkins, Ph.D. thesis, Lehigh University, 1990; R. D.
Jenkins, C. A. Silebi, and M. S. El-Aasser, in Polymers as Rhbeology Modifiers, edited by D. N. Schulz and J. E. Glass, American Chemical Society Symposium Series No. 462 (American Chemical Society, Washington, D.C., 1991), p. 223.

[12] T. Annable, R. Buscall, R. Ettelaie, and D. Whittlestone, J. Rheol. 37, 695 (1993).

[13] R. D. Jenkins (private communication).

[14] Ljubinko Kondic, Peter Palffy-Muhoray, and Michael J. Shelley, Phys. Rev. E 54, R4536 (1996).

[15] M. Kawaguchi, A. Shibata, K. Shimomoto, and T. Kato, Phys. Rev. E 58, 785 (1998).

[16] See, e.g., R. G. Larson, Constitutive Equations for Polymer Melts and Solutions (Butterworths, London, 1988).

[17] D. S. Malkus, J. A. Nohel, and B. J. Plohr, J. Comput. Phys. 87, 464 (1990).

[18] G. Vinogradov et al., J. Polym. Sci., Part A-2 10, 1061 (1972).

[19] D. Bonn, H. Kellay, M. Ben Amar, and J. Meunier, Phys. Rev. Lett. 75, 2132 (1995).

[20] D. H. Vlad and J. V. Maher (unpublished).

[21] R. B. Bird, R. C. Armstrong, and O. Hassager, Dynamics of Polymeric Liquids (Wiley, New York, 1987), Vol. 1.

[22] S. D. R. Wilson, J. Fluid Mech. 220, 413 (1990); J. S. Ro and G. M. Homsy, J. Non-Newtonian Fluid Mech. 57, 203 (1995); D. Bonn and J. Meunier, Phys. Rev. Lett. 79, 2662 (1997).

[23] J. W. Cahn and J. E. Hilliard, J. Chem. Phys. 28, 258 (1958); H. T. Davis, in Numerical Simulation and Oil Recovery, edited by M. Wheeler (Springer-Verlag, Berlin, 1988), p. 105; S. E. May and J. V. Maher, Phys. Rev. Lett. 67, 2013 (1991); D. H. Vlad and J. V. Maher, Phys. Rev. E 59, 476 (1999). 